\title{
REFORMA SEXUAL, CONTROL DE NATALIDAD, NATURISMO Y PACIFISMO. LA CULTURA LIBERTARIA TRASATLÁNTICA EN LAS DÉCADAS DE 1920 Y 1930: ESTUDIOS. REVISTA ECLÉCTICA (1928-1937) Y SU PROYECCIÓN Y REDES EN AMÉRICA
}

Sexual reform, birth control, naturism and pacifism. The Transatlantic libertarian culture in 1920 and 1930 decades. Estudios. Revista Ecléctica (1928-1937): Projection and networks in the Americas

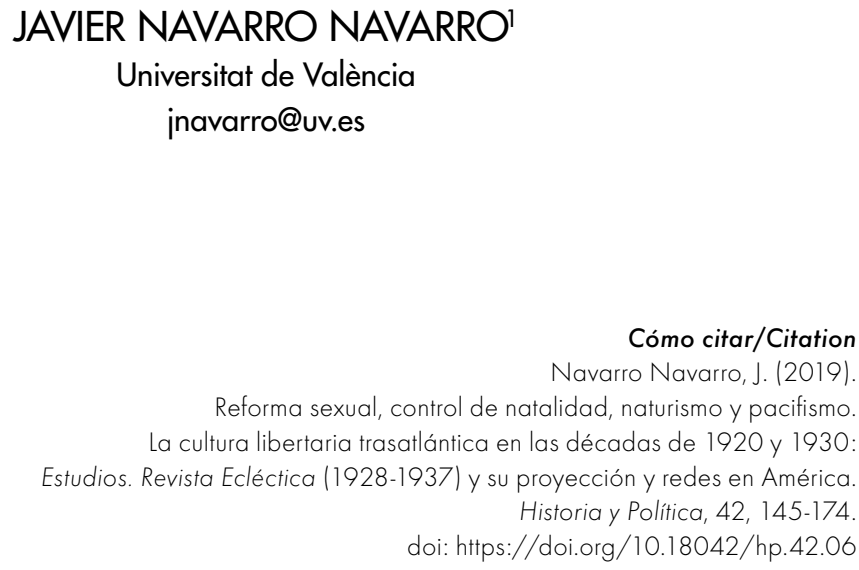

\section{Resumen}

Los redactores y principales colaboradores de la influyente revista cultural libertaria española Estudios (Valencia, 1928-1937) mantuvieron a lo largo de la vida

\footnotetext{
1 El autor pertenece al Grup d'Estudis Històrics sobre les Transicions i la Democràcia (GEHTID), que es un grupo de investigación de excelencia PROMETEO de la Conselleria d'Educació de la Generalitat Valenciana (GVPROMETEO2016-108).
} 
de la publicación un interés permanente por conectarla, no solo con las principales redes del anarquismo global de entreguerras, sino también con campańas internacionales de aquellos ańos como la lucha por la difusión del control de natalidad y la eugenesia, la reforma sexual, el naturismo o el pacifismo. En este sentido, y continuando la labor de su predecesora, Generación Consciente (1923-1928), Estudios se vinculó a conocidos militantes, escritores, científicos y propagandistas en general de estas ideas y prácticas en Europa y América. A través de la propia revista, los contactos personales con estos individuos y su intensa labor publicística (con un activo servicio de librería y una muy fecunda actividad editorial), Estudios tejió redes a lo largo de estos ańos que contribuyeron a la difusión de esta nueva cultura anarquista internacional del período de entreguerras. América resultó aquí clave, tanto por la importancia que para la revista tuvo su distribución y venta en diversos países americanos, como por los vínculos que se establecieron con militantes, grupos, editoriales, etc., de aquel continente, afines de una u otra manera a las orientaciones ideológicas y culturales de Estudios.

\title{
Palabras clave
}

Anarquismo; cultura libertaria; neomaltusianismo; naturismo; pacifismo.

\begin{abstract}
The editors and main collaborators to the influential Spanish libertarian magazine Estudios (Valencia, 1928-1937) showed a permanent interest in connecting the magazine with the main networks of global anarchism in the interwar years, and also with contemporary international campaigns in defense of birth control, eugenics, sexual reform, naturism or pacifism. As its predecessor did-the magazine Generación Consciente (1923-1928) — Estudios was linked to well-known militants, writers, scientists and propagandists in general of those ideas and practices in Europe and America. Estudios built networks using the magazine itself, its personal contacts with those individuals, and also an active book sale service and an intense publishing work. Therefore, Estudios contributed to the diffusion of this new international anarchist culture of the interwar period. The Americas were crucial here, due to their financial importance for the magazine in terms of sales, but also because of the links established with militants, groups, publishers, etc., of that continent that shared the ideological and cultural orientations of Estudios.
\end{abstract}

\section{Keywords}

Anarchism; libertarian culture; neomalthusianism; naturism; pacifism. 


\section{SUMARIO}

I. GENERACIÓN CONSCIENTE Y ESTUDIOS, EMPRESA SINGULAR. II. ESTUDIOS Y AMÉRICA: UN PÚBLICO LECTOR. III. ESCRITORES/AS Y ACTIVISTAS. IV. REDES EDITORIALES. V. CONCLUSIONES. BIBLIOGRAFÍA.

La cultura y la educación ocuparon siempre un rol central en la teoría y la práctica del movimiento libertario español como premisas revolucionarias y parte integrante de su proyecto emancipador ${ }^{2}$. En este sentido, la prensa ácrata desempeñó un papel fundamental como medio propagandístico y de combate, canal de información y comunicación interno, y forma de articulación y cohesión orgánicas. Pero también como herramienta de difusión y debate cultural. Destacó aquí la publicación de revistas de carácter específicamente cultural, tarea a la que se dedicaron importantes esfuerzos. A títulos ya presentes desde finales del siglo XIX como Ciencia Social, Acracia, Natura o La Revista Blanca - esta última con una segunda época desde 1923 - se sumarían a partir de la década de 1920 numerosas cabeceras: Iniciales, Ética, Orto, Generación Consciente-Estudios, Tiempos Nuevos, etc.

La presente contribución se centra en la revista Estudios, editada en Valencia entre 1928 y 1937 y heredera de la publicación alcoyana Generación Consciente (1923-1928). En sus páginas tuvo cabida la reflexión social y económica — más que el análisis de la actualidad política inmediata- y en particular los temas culturales de carácter general (desde la geografía y la historia al arte, literatura, ciencia, pedagogía, etc.), aunque fue su énfasis en cuestiones como la reforma sexual, el control de natalidad, la eugenesia y, también en menor medida, el naturismo o la medicina alternativa, su rasgo más distintivo. En Estudios no abundaban los mensajes explícitamente propagandísticos (aunque sí la apuesta por la transformación social de carácter revolucionario), con una definición pretendidamente "ecléctica», como rezaba su cabecera, y una adscripción libertaria muy general, que conectaba asimismo con temas propios del mundo radical o librepensador, como el anticlericalismo, o con el pacifismo del período de entreguerras, otro leit motiv de la publicación.

Estudios alcanzó un gran éxito de difusión y prestigio en los medios obreristas españoles. Analizaremos en primer lugar qué razones pueden ayudar a

2 Álvarez Junco (1991): 73; Litvak (1981): XV, y Navarro Navarro (2010). 
explicar este fenómeno y en qué consistió la singularidad y personalidad propias de la revista que la hicieron reconocible para su público lector.

Pero Estudios tuvo asimismo una significativa presencia internacional y en especial un fuerte vínculo con el continente americano en las décadas de 1920 y 1930, donde fue ampliamente distribuida y tuvo un gran número de lectores. La difusión, prestigio y éxito mencionados lo fueron también entre los libertarios, el movimiento obrero en general y los medios librepensadores a ambos lados del Atlántico.

La tesis que aquí defendemos es que a partir de su cobertura de un amplio abanico de temas culturales de especial interés en aquellos años entre estos sectores (como el neomaltusianismo, la eugenesia, la reforma sexual, el naturismo, las variadas propuestas de transformación revolucionaria de la cotidianeidad o el pacifismo), pero también con la crítica global al capitalismo y la reflexión sobre la futura sociedad postrevolucionaria a cargo de autores ampliamente reconocidos de impronta libertaria, revolucionaria o progresista, Estudios tejió una red transnacional —y específicamente transatlántica- que conectó militantes, escritores, científicos, médicos (cuyo papel resultó aquí fundamental) y propagandistas en torno a todas estas cuestiones en las páginas de la revista y en los libros y folletos de su editorial, así como una amplia masa de lectores que compartían esas afinidades progresistas y revolucionarias.

América fue una de las principales áreas de difusión de la revista (lo que sirvió para garantizar, entre otras, cosas su supervivencia gracias a esas ventas), y escritores de varios países americanos colaboraron regularmente con ella desde allí, como veremos (Carlos Brandt, María Lacerda de Moura, Juan Lazarte o Alfonso L. Herrera, por citar solo algunos). Asimismo, se articuló un flujo cultural de intercambio y circulación de información y de ideas a través del Atlántico que se consolidó con la vinculación de Estudios con otras publicaciones culturales similares o editoriales en el continente americano. Pretendemos demostrar que esta vinculación y esos flujos, lejos de ser secundarios, fueron esenciales para la revista y también para el proyecto transnacional de difusión de esas ideas y prácticas que Estudios impulsaba ${ }^{3}$.

3 Este artículo se beneficia y pretende inscribirse también en la línea de trabajos que han utilizado la perspectiva transnacional para una comprensión más profunda de los movimientos sociales y las culturas políticas más allá del marco nacional, de sus redes y formas de circulación de personas, ideas, elementos culturales, etc., en particular en el caso del anarquismo, donde viene mostrándose especialmente útil y fecunda en ańos recientes. Hay ya al respecto una significativa bibliografía. Un ejemplo, con una reflexión en torno a la pertinencia de este enfoque en el estudio del anarquismo, en: Sueiro Seoane (2013). 


\section{GENERACIÓN CONSCIENTE Y ESTUDIOS, EMPRESA SINGULAR}

Estudios. Revista Ecléctica, publicada en Valencia mensualmente entre diciembre de 1928 y junio de $1937^{4}$, fue en realidad la continuación natural de otra revista, Generación Consciente (también subtitulada Revista Ecléctica), que había nacido en Alcoi en 1923. El cambio lo fue solo en lo que se refiere al título de su cabecera. Al elegir una denominación deliberadamente neutra y aséptica, Estudios, sus responsables trataron de esquivar la censura y la persecución legal de la que fueron objeto a partir de 1928 los medios partidarios del control de natalidad por parte de la Dictadura de Primo de Rivera.

Generación Consciente, surgida a su vez del semanario cenetista alcoyano Redención (órgano del Sindicato Único de Trabajadores de Alcoy y portavoz de la CNT, aparecido en 1921), y cuya redacción se trasladó en 1925 a Valencia, se caracterizó por su defensa de la «maternidad consciente», el control de la natalidad obrera y la regeneración física del proletariado (con una selección racional de su reproducción que garantizara unas óptimas condiciones de vida y salud), así como por la difusión de una nueva moral sexual alternativa a la tradicional y hegemónica ${ }^{5}$. La reflexión sobre el rol social de las mujeres, así como el naturismo y la medicina natural fueron también otros de los temas habituales de la publicación, de la misma manera que ocurriría con Estudios. Esta continuaría asimismo con ese modelo de revista teórica, cultural y de pensamiento, pero sin referencias concretas al momento político que se vivía, que había permitido a Generación Consciente sobrevivir durante la Dictadura.

Lo cierto es que Generación Consciente y Estudios alcanzaron una gran difusión (por encima sin duda de otras publicaciones de similar corte ideológico $^{6}$ ) y una amplia distribución en todo el territorio peninsular, así como también por diversos países de Europa y América, como veremos. Aparte de sus ventas, es evidente la repercusión de las revistas en el mundo social y cultural libertario espańol, y por extensión en el obrerismo y los sectores

$4 \quad$ El análisis de la revista en Navarro Navarro (1997a). Una reproducción facsímil de la publicación en CD, en Estudios. Revista Ecléctica. Números 64-165. Valencia, 19281937 (2007).

5 Sobre Generación Consciente, Navarro Monerris (1988) y Navarro Navarro (1997b).

6 Aunque se ha hablado en algún momento de cifras de 70000 ejemplares para alguno de sus números (tal vez en referencia a los extraordinarios anuales, los más vendidos), esta cifra resulta sin duda exagerada. Evidentemente, su tirada media sería menor; en el mejor de los casos tal vez inferior a 50000 ejemplares, y más probablemente, como seńalaba el Delegado de la AIT, Alexander Shapiro, de unos 25000 a la altura de 1933. Shapiro (1933): 492. 
populares afines a esas actitudes y temáticas durante la década de los treinta. Su prestigio fue indudable en el conjunto del anarcosindicalismo de esos años: la imagen de calidad y referencia de autoridad de la publicación ${ }^{7}$ hacía que fuera comentada y sus artículos citados con frecuencia en periódicos y revistas afines.

Más allá de su impacto contemporáneo, lo cierto es que la revista dejó huella en muchos de sus lectores, como muestran los testimonios de antiguos militantes libertarios, que refieren a menudo la influencia que Estudios ejerció sobre ellos, aunque esta no se circunscribía al mundo ácrata ${ }^{8}$. Uno de los sectores más permeables a esta influencia fue la juventud obrera más inquieta, generalmente autodidacta, anhelante de cambios en los comportamientos cotidianos y, entre ellos, en la actitud ante la «cuestión sexual», ampliamente debatida en esos años.

Tal como nos interrogábamos antes, ¿en qué residió, por tanto, la singularidad de Generación Consciente y Estudios, que las hizo reconocibles en el panorama obrerista en España y también en el mundo libertario y librepensador hispanohablante de las décadas de 1920 y 1930? Y, paralelamente, ¿qué razones ayudan a explicar el éxito de este proyecto editorial a ambos lados del Atlántico? En mi opinión, estas radicaron por un lado en la capacidad para conectar con temáticas de amplio seguimiento y que estaban, además, presentes en el debate público internacional durante el período de entreguerras. Por otro lado, este aspecto fue reforzado por las acertadas estrategias editoriales de la propia revista y también por su vocación global. Todos estos elementos consolidaron a su vez el carácter transnacional de Estudios y su capacidad para constituir unas redes que fueron más allá del marco nacional.

Sin duda, un factor fundamental fue, como venimos comentando, la especialización de la revista en temas como la defensa del neomaltusianismo y el control de natalidad, la eugenesia y la «reforma sexual», a los que Estudios

7 En muchas ocasiones nos referiremos en singular a las dos publicaciones, ya que, en realidad, se trata de una misma revista que cambia de título.

8 En uno de estos testimonios se señala: «[...] su influencia iba mucho más allá del tiraje, por cuanto los temas que en la Revista se planteaban eran motivo de discusiones continuas, en conversaciones personales o en círculos más o menos numerosos, interesados ya fuese por uno u otro de los temas planteados. La variedad de los círculos era tal, que de este hecho resultaba aun mayor la difusión de la Revista: Naturistas, vegetarianos, esperantistas, liberales, reformistas o revolucionarios, y así podríamos seguir la enumeración [...]». Testimonio de Manuel Llatser al autor, mayo 1995. Para testimonios de antiguos lectores de la publicación en general, véase Navarro Navarro (1997a): 74-82 
dedicó una atención preferente, los que le dotaron de una identidad reconocible y que fueron, en buena medida, clave de su éxito. La publicación defendía la reducción de la natalidad obrera y la mejora de la calidad de la descendencia (eugenesia), con un discurso de impronta higienista y regeneracionista, al tiempo que se incidía en una nueva concepción laica y libre de la sexualidad, desvinculada de la procreación.

Ello conectaba a Estudios con toda una tradición libertaria previa de defensa del neomaltusianismo (que se acompañaba a su vez de la difusión de las distintas prácticas anticonceptivas y el debate en torno a ellas) desde los tiempos de la aparición de la sección española de la Liga Universal Neomalthusiana en 1904 y la labor de divulgación editorial de Luis Bulffi en Barcelona a partir de entonces desde su órgano Saludy Fuerza. También la vinculaba con los discursos y actitudes en torno a la eugenesia que se habían ido fraguando en los medios ácratas en años anteriores, o con la opinión de determinados sectores libertarios a la hora de entender la sexualidad y su crítica a la cultura imperante en este ámbito y a la sexología institucional, aspectos que veremos ampliamente desarrollados en la publicación?. Sin duda, Generación Consciente y Estudios destacaron especialmente por su capacidad para colocar estos debates en el centro de atención, mostrando el punto de maduración de estos en determinados medios del anarquismo español y contribuyendo sin duda a su difusión.

Uno de los objetivos prioritarios de sus redactores fue, precisamente, la divulgación de estos principios entre el público popular y trabajador. También la potenciación del debate en torno a estas ideas en el movimiento obrero español, desde aquellos sectores libertarios más avanzados y sensibles a estas cuestiones y a los que la revista representaba ${ }^{10}$. Puede afirmarse que Generación

9 Por razones de extensión, no podemos entrar aquí en detalle en el análisis de estos debates en el seno del anarquismo español del primer tercio del siglo xx ni en mayor profundidad sobre los contenidos concretos de estos discursos, actitudes y prácticas. Sobre todo ello hay ya una significativa bibliografía. Destacan los trabajos de Mary Nash. Entre ellos Nash (1995 y 1999). Asimismo, resultan esenciales en un sentido global (desde el neomaltusianismo y la eugenesia a la sexualidad en general) las investigaciones y obras de Richard Cleminson (2008). Para el neomaltusianismo ibérico, en general, y también específicamente anarquista, es fundamental asimismo la lectura de los trabajos de Eduard Masjuan (2000). En lo relativo a la eugenesia en estos años Álvarez Peláez (1995). Desde la perspectiva del anarcoindividualismo Díez (2007).

10 El médico libertario alavés Isaac Puente, uno de los principales colaboradores de la revista y auténtico portavoz de la publicación en estos temas, resumía en junio de 1931 los principales puntos del "programa» de Estudios en este ámbito: 
Consciente y Estudios se convirtieron en unas de las principales publicaciones españolas en el ámbito de la difusión de la teoría y la práctica de las doctrinas neomaltusianas y eugenésicas. Fue frecuente en sus páginas el análisis de las características de los distintos métodos anticonceptivos, así como también los anuncios de su distribución y venta por correspondencia. Junto a estos temas centrales, cabe ańadir la apuesta por el naturismo y la medicina alternativa, con su difusión a través de múltiples vías, como veremos, por ejemplo, mediante los consultorios médicos abiertos a los lectores (al igual que sucedió con la sexualidad).

Como señalamos, las estrategias editoriales de la revista fueron asimismo fundamentales para reforzar esta difusión y proyección popular. Desde los inicios de Generación Consciente, sus responsables pusieron en marcha un servicio de distribución de libros y folletos de otras casas editoriales más o menos afines: centenares de títulos (ya unos 2000 a la altura de 1929) que la revista ponía en venta - la Biblioteca de Estudios- a través de un catálogo anunciado en sus páginas. Un factor que contribuyó al éxito de este servicio fue el perfil de las obras vendidas, bastante ecléctico, con una temática en la que primaba lo cultural y lo literario sobre las obras de carácter doctrinal, así como un enfoque en absoluto exclusivamente anarquista, con amplia presencia de títulos con un enfoque obrerista en general, librepensador, republicano o simplemente progresista.

Asimismo, Generación Consciente y Estudios contaron con una editorial propia en la que aparecieron más de un centenar de títulos entre libros y folletos entre 1923 y 1937. Tanto la Biblioteca de Estudios como la editorial permitían atender los objetivos educativos y propagandísticos de la publicación, pero también sirvieron asimismo para garantizar con sus ventas su supervivencia. También en la editorial Estudios primó un criterio amplio y ecléctico. No faltaron temas sociales y políticos desde una perspectiva crítica y revolucionaria, o el pensamiento en general y la literatura, pero lo cierto es que las más conocidas colecciones de la editorial se centraron en cuestiones como la divulgación sexual, el rol social de las mujeres o el debate sobre la anticoncepción — todas de gran seguimiento en estos años—, así como en otros temas también de aplicación práctica en la vida diaria, como la medicina, el naturismo o la formación cultural en general para autodidactas.

educación sexual; abolición de la prostitución (en primer lugar, de su reglamentación por el Estado); lucha antivenérea y difusión de medios para prevenir este tipo de enfermedades; libertad sexual de la mujer; control de la natalidad, y «desintoxicación religiosa del sexo». Un médico rural, "A modo de programa», Estudios, 94, junio 1931. 
Esta variedad y la capacidad para conectar con esos temas «de avanzada» en esos años explican el éxito popular de estas ediciones, junto con una oferta diversa y una presentación muy atractiva. Muchos de estos libros tenían vistosas cubiertas, portadas e ilustraciones de destacados cartelistas y grafistas como Josep Renau o Manuel Monleón, con abundancia de color y representaciones de figuras desnudas para los títulos sobre tema sexual. Destaca también la apuesta de la editorial por lanzar colecciones de folletos y pequeños libritos a precios reducidos y con títulos como: Ayer, hoy y mañana, Conocimientos útiles de medicina natural o Antología de la felicidad conyugal (conocimientos útiles para la vida privada). Esta activa y continuada labor en el campo de la distribución y edición de libros cimentó el éxito y el prestigio de Estudios dentro y fuera de las fronteras españolas, con tanta intensidad como la propia revista.

Por otro lado, cabe mencionar otro apartado de la publicación con un importante seguimiento popular entre sus lectores, también heredado de los tiempos de Generación Consciente: los llamados "consultorios médicos». Además de la publicidad de las consultas particulares que médicos obreristas publicitaban en sus páginas para atender al público trabajador a precios reducidos o de forma gratuita, estos se prestaron también a responder a las preguntas y dudas de los lectores de Estudios, con secciones permanentes cuyo fin era orientar y divulgar sobre una amplia variedad de cuestiones. No fue este tampoco un rasgo exclusivo de Estudios (otras publicaciones ácratas similares, como La Revista Blanca o Iniciales, dispusieron asimismo de ellos), pero los consultorios abiertos por aquella gozaron de un gran prestigio que perduró posteriormente en la memoria de sus lectores. Fueron bien de carácter médico en general, bien relacionado en particular con la sexualidad — como el célebre «Consultorio psíquico-sexual» que el psiquiatra libertario catalán Félix Martí Ibáñez comenzó en enero de 1936 en la revista ${ }^{11}$ — o bien con una orientación naturista, como el consultorio del doctor Roberto Remartínez («Preguntas y respuestas»), otro habitual colaborador de la publicación. También se puso en marcha un consultorio de carácter científico-técnico, con preguntas sobre materias variadas en este ámbito y mantenido por el ingeniero y escritor anarquista Alfonso Martínez Rizo.

Entre las estrategias editoriales de Estudios, una especialmente acertada —además de su precio asequible y su temática y contenido variados (y en especial, como ya dijimos, en todo lo relacionado con la sexualidad) — fue, como apuntábamos, lo cuidado y llamativo de su presentación gráfica. Con la llegada de la República, y en especial a partir de 1932, las portadas de los

11 Una antología, en Martí Ibáñez (1975). 
números de la revista se hicieron más atractivas y explícitas, tanto en lo político como también en la exhibición del desnudo femenino. Tuvo aquí un papel clave la colaboración de dos artistas de la talla de los ya mencionados, Josep Renau y Manuel Monleón, que confeccionaron muchas de esas portadas (y numerosas ilustraciones y fotomontajes interiores) y también las de varios de los libros de su catálogo. Aquellas se llenaron ahora de color y fotomontajes y composiciones muy llamativas. Algunas eran abiertamente ideológicas y — de forma creciente a lo largo de la década de los treinta— de significación antifascista. Pero también incluían a menudo la representación de figuras femeninas desnudas (también en muchos de los libros y folletos editados por Estudios). Estas, sin duda, constituían una buena alegoría de los ideales naturistas y regeneracionistas de la revista, pero ayudaban también de paso a atraer lectores y coqueteaban - pese a las airadas declaraciones en contra de los responsables de Estudios - con la publicística erótica y pornográfica de la época, al igual que sucedía con muchos de los libros de su editorial ${ }^{12}$.

Por último, parte del éxito y el prestigio de la revista se debió a que supo mantener, dentro de su orientación ideológica específica, su carácter relativamente ecléctico, como rezaba su subtítulo. Fue fundamental que no se tratara de una publicación orgánica para conectar así de manera amplia con el mundo obrerista, librepensador y progresista desde una perspectiva culturalista. Aunque genéricamente libertaria, doctrinalmente obrerista y revolucionaria en sus declaraciones y editoriales, así como declaradamente antifascista en el panorama internacional a lo largo de la década de los treinta ${ }^{13}$, la revista supo mantenerse al margen de las disputas internas que vivió el movimiento libertario español en estos ańos, sin adscribirse a una tendencia concreta. Todo un éxito estratégico, apelando siempre a su orientación formativa. Por otro lado, Estudios contaba con grupos de lectores que incluían también militantes obreros de otras opciones políticas, librepensadores, progresistas, republicanos, naturistas, pacifistas, feministas, partidarios del neomaltusianismo y, por qué no, individuos sin vocación política o ideológica concreta, pero interesados por sus opiniones - o por sus dibujos o fotografías de desnudos, como apuntábamos más arriba- sobre el tema sexual.

12 Álvarez Peláez (2011) y Guereña (2013), especialmente el capítulo 1: «Le sexe devoilé. Les collections populaires d'éducation sexuelle».

13 El contexto internacional fue despertando cada vez mayor interés, con temas como la crisis internacional del capitalismo, la guerra chino-japonesa y, sobre todo, el auge del nazismo. La revista trató de alertar desde sus páginas sobre los avances del fascismo (llamando a la unidad antifascista) y la preparación de una próxima guerra. 


\section{ESTUDIOS Y AMÉRICA: UN PÚBLICO LECTOR}

Estudios tenía "corresponsales administrativos» en las siguientes ciudades: Barcelona, Madrid, Sevilla y Granada (España), Buenos Aires y Rosario-Santa Fe (Argentina), Montevideo y Salto (Uruguay), Camagüey (Cuba), Maracaibo (Venezuela) y San José (Costa Rica). La publicación siempre gozó de una importante aceptación fuera de las fronteras españolas, especialmente en América, desde los tiempos de Generación Consciente, que ya había establecido a la altura de 1928 diecinueve puntos de distribución fuera de España, cubiertos por enlaces establecidos en Islas Canarias, Francia, Argentina, Brasil, Chile, Cuba, Estados Unidos, Méjico, Puerto Rico, Perú, Colombia y Paraguay. Francia y Bélgica fueron los destinos principales de la revista en Europa. Sin embargo, América fue un ámbito de expansión de Estudios mucho más evidente y sensible para esta que otros países europeos, incluyendo Francia o Portugal. Significativamente, el precio de suscripción anual de la publicación tanto para Espańa y Portugal como para América era el mismo (6,50 pesetas), mientras que para los demás países europeos era de 8 . Ya en febrero de 1929 (número 66), la revista señalaba que tenía varias demandas de ejemplares de su número extraordinario-almanaque del mes anterior, «destinados a la venta en kioscos y librerías de Sudamérica».

Estudios contó con un buen número de lectores en América. En alguna ocasión se hablado de más de 20000 ejemplares distribuidos solo en Argentina ${ }^{14}$. Más allá de la obvia exageración de esta cifra, proveniente de un testimonio militante posterior, lo cierto es que este país aparece repetidamente mencionado en las notas administrativas de la revista y las referencias concretas sobre Argentina superan en ocasiones a las de muchas provincias españolas. Asimismo, un buen termómetro de la recepción de Estudios en América lo constituye, por ejemplo, la lista de «paqueteros morosos», es decir, vendedores de la revista en puntos alejados de su lugar de origen -individuos particulares, establecimientos, asociaciones, etc.- que no liquidaban de vuelta el producto de las ventas de sus ejemplares (o libros y folletos de sus catálogos), generando por tanto una deuda con Estudios. La revista se quejaba continuamente de esta circunstancia, que explicaba buena parte de su déficit, así como de estos individuos «desaprensivos». A la altura de noviembre de 1933, la cantidad que adeudaban estos paqueteros ascendía ya a 7194,30 pesetas, y la administración de la revista decidió publicar en sus páginas un listado con sus nombres. La lista es indicadora de la difusión geográfica de Estudios por

14 «Sólo para la Argentina salían veintitantos mil ejemplares [...]», Cano Carrillo (1972): 5699. 
España, Europa, norte de África y América. En particular denota la expansión de la revista por este último continente, muy por encima de su difusión en cualquier otro país europeo, incluyendo Portugal y Francia. Veamos en concreto las referencias a estos «morosos» en América (con la cifra adeudada en pesetas al lado):

AREQUIPA (Perú, S. A.), Armando Rivera, librería (108,55)

BUENOS AIRES (Argentina), Antonio Almadén, librería (21)

BUENOS AIRES (Argentina), Emilio A. Alvarez $(25,55)$

BUENOS AIRES (Argentina), José Coma $(63,50)$

BUENOS AIRES (Argentina), Eugenio Navas $(255,05)$

GENERAL PICO (Argentina), Isidro D. Martínez (63)

GENERAL PICO (Argentina), Juan Ferrini $(151,40)$

HOMESTEAD (USA), Juan Bais Ayala $(79,65)$

LOS ANGELES (USA), Lorenzo Hernández $(215,95)$

LOS ANGELES (USA), M. Flores Cabanillas (241)

JAUJA (Perú, S. A.), Máximo Pecho, librería $(96,65)$

LIMA (Perú, S. A.), Agencia Geo $(102,95)$

MÉJICO (Centro América), Martín Rodó, librería $(59,60)$

NEW YORK (USA), Librería Cervantes (16)

NEW YORK (USA), J. A. Pérez, Librería Intuición $(85,70)$

PUERTO MAR DEL PLATA (Argentina), José Ujaldón $(34,90)$

QUITO (Ecuador, C. A.), Luis F. Torres, Librería Horizontes $(88,25)$

ROSARIO (Argentina), L. Gornotti Eyzaguirre (59)

ROSARIO (Argentina), Ulpiano Pérez $(83,85)$

ROSARIO (Argentina), Bernabé Villena, G. Pro Prensa $(95,25)$

SAN JUAN (Argentina), Saturio Pina $(31,30)$

SAN JUAN (Argentina), Bautista Platero $(59,40)$

SAN PEDRO (Argentina), Vicente Perrone $(41,50)$

SANTIAGO (Chile, S. A.), Quiterio Chávez Utrera, librería (79'25)

SAO PAULO (Brasil. S. A.), Francisco Aroca (182’90)

SAO PAULO (Brasil, S. A.), Miguel Collado López (28'90)

SHELBY HUARON (Perú, S. A.), Edilberto Párragá (18)

TRES ARROYOS (Argentina), Domingo Labourcade $(106,70)$

TUCUMÁN (Argentina), Gregorio F. Fernández $(103,45)^{15}$

15 «A los lectores y amigos de Estudios [...]. Lista de Morosos», Estudios, 123, noviembre 1933. Cuatro meses después, en el número 127 de la revista (marzo 1934), se ańadió también a la lista la población argentina de Lanús, señalando la deuda correspondiente a la «Biblioteca Popular» de esa localidad (69,70 pesetas). 
Aparte de los particulares, aparece en esta lista una red de librerías en distintos puntos de América, así como algún grupo "pro-prensa». En cuanto a la distribución geográfica, destaca sin duda Argentina, en particular Buenos Aires (con varios deudores y elevadas cantidades), pero también de manera significativa Rosario, así como General Pico y San Juan, y en menor medida Tucumán, Mar del Plata, San Pedro y Tres Arroyos. La preponderancia de Argentina en esta lista es incuestionable, pero también figuran seguidamente los Estados Unidos —Nueva York, Los Ángeles, Homestead (Florida)—. Sobresale aquí la abultada deuda de los paqueteros de Los Ángeles (entre algunas de las más altas de esta amplia lista en general), así como la presencia de dos librerías de Nueva York entre los deudores: Cervantes e Intuición. A continuación encontramos también referencias a Perú (Lima, Arequipa, Jauja, Shelby Huarón), Brasil (Sao Paulo), México (Ciudad de México), Chile (Santiago) y Ecuador (Quito).

Asimismo, hallamos huellas de los lectores americanos de Estudios en los consultorios abiertos en sus páginas (de carácter médico o científico) a lo largo de la vida de la revista. Especialmente significativa resulta también la creación de una "peña» de Estudios en Argentina, concretamente en la Pampa, en General Pico, en los inicios de $1936^{16}$.

La distribución de la revista en América no estuvo, sin embargo, exenta de obstáculos. Y no solamente por su orientación ideológica libertaria. Su especialización temática en el ámbito de la reforma sexual, el control de natalidad, la eugenesia o el naturismo, además de la ya mencionada utilización iconográfica del desnudo femenino y/o nudismo, le ocasionaron problemas añadidos. En enero de 1937 se protestaba desde las páginas de la revista por la prohibición por parte de los Gobiernos de Cuba y Argentina de la entrada a sus países de ejemplares de Estudios bajo la acusación de publicación "pornográfica», tras lo cual los responsables de la revista veían un pretexto bajo el que se escondían las escasas simpatías de esos Gobiernos hacia los antifascistas republicanos españoles en el contexto de la guerra civil:

PROTESTAMOS: Hace ya varios meses se prohibió la entrada de ESTUDIOS en Cuba con el pretexto de que era pornográfica. No hicimos caso entonces, considerando que esperar un poco de juicio o sentido común del émulo del dictadorzuelo Batista, que en la Administración de Correos de La Habana ejerce sus funciones, era pedir peras a un olmo. Fue sometida nuestra Revista al fallo imparcial de una comisión de médicos y periodistas cubanos, quienes dictaminaron que las páginas de ESTUDIOS contenían artículos de

16 Estudios, 150, febrero 1936. 
carácter científico de gran utilidad para la vida sexual y privada, pero que en ninguna forma podían considerarse pornográficos ni inmorales. A pesar de lo cual, el pingüino administrador o funcionario que dio la orden de prohibición no ha querido anular su draconiana disposición.

Pero ahora nos llega la noticia de que el Gobierno argentino se ha incautado de todos los paquetes destinados a nuestro corresponsal en Buenos Aires, callándose los motivos, que no son otros que su fobia antiespañola, de acuerdo con sus amos los jerifaltes fascistas de Italia y Alemania.

Y nuestro cónsul en Buenos Aires..., bien, gracias ${ }^{17}$.

\section{ESCRITORES/AS Y ACTIVISTAS}

Pero la proyección y vinculación americana de Estudios fue más allá de esta constatación de su distribución y lectura en América. Y en varios sentidos. Por un lado, la revista siempre manifestó un vivo interés por lo acontecido al otro lado del Atlántico, tanto en una dimensión política y social como en lo cultural e intelectual. Algunos de los principales redactores de Estudios, entre ellos el conocido militante libertario español Antonio García Birlán, encargado de secciones fijas como "Actualidad», «Gacetilla» o "Autores y libros» (bajo seudónimos como Dionysios o Julio Barco), comentaban habitualmente y ponían al día a los lectores sobre temas relacionados con América. Otro conocido escritor anarquista español, Manuel Costa Iscar (seudónimo de Manuel Faciabén Esquer), afincado de manera permanente en Argentina desde la década de los veinte, mantuvo una sección denominada precisamente "Cartas de América».

Caso similar a este es el de otro militante, escritor e intelectual ácrata, Campio Carpio (a su vez seudónimo de Campio Pérez Pérez, de origen gallego y que también ejerció su actividad desde los años veinte en Argentina, donde viviría hasta su muerte), asimismo colaborador de Estudios. También puede incluirse aquí al médico naturista libertario valenciano José María Martínez Novella, que residió y desarrolló su carrera profesional en Estados Unidos también desde la década de los veinte, en especial en New Jersey y Nueva York, y desde donde escribía habitualmente en Estudios sobre naturismo, reforma sexual, medicina, biología y ciencia en general. Martínez Novella se hizo cargo por ejemplo del consultorio científico de la revista titulado «Al día con la ciencia» durante los meses en que el titular de esta

17 Estudios, 160, enero 1937. 
sección, el ingeniero anarquista Alfonso Martínez Rizo, marchó al frente durante la guerra civil ${ }^{18}$.

Por otro lado, numerosos militantes libertarios, obreristas en general o librepensadores americanos, colaboraban de forma habitual en Estudios. Se trata de médicos, científicos, escritores o simplemente propagandistas muy conocidos, y que también contribuyeron a la difusión de Estudios en América. La lista es muy amplia. Podemos mencionar, por ejemplo, entre los médicos y científicos (que escribieron sobre temas de especial interés para la publicación, como los relacionados con el control de la natalidad, la sexualidad o el naturismo, pero también sobre la divulgación médica o científica en general) a los mexicanos Alfonso L. Herrera y Carlos López de Gabriel (médico homeópata), o a los argentinos Juan Lazarte y Enrique Feinmann. Entre los escritores y propagandistas anarquistas puede citarse, entre otros, a los argentinos Alberto Ghiraldo, ya asiduo colaborador de Generación Consciente (y cuya novela Humano Ardor fue ampliamente publicitada por Estudios), Julio R. Barcos, Rodolfo González Pacheco (escritor y dramaturgo cuya obra Carteles también fue difundida y elogiosamente comentada desde la publicación) y el radical Alcides Greca; o el venezolano Carlos Brandt. Todos ellos escribieron en Estudios sobre múltiples temas, y vieron también anunciados allí sus libros y folletos, publicados algunos de ellos desde la propia editorial de la revista.

Asimismo, el conocido anarquista francés Gaston Leval, que desde mediados de la década de los veinte residió en Argentina y Uruguay, era un asiduo colaborador de Estudios en estos años. Leval escribió desde Rosario en noviembre de 1930 (número 87 ) ofreciendo originales de algunos de sus libros a las editoriales afines, entre ellos su Infancia en cruz (narración autobiográfica), que finalmente publicaría en 1933 la misma editorial Estudios.

Nos detendremos brevemente en algunos de estos nombres, por su dilatada colaboración con Estudios o por la influencia y eco que tuvieron a través de sus páginas en algunos temas clave de la publicación. Sin duda, merece destacarse en primer lugar el caso de la escritora libertaria brasileña Maria Lacerda de Moura (1887-1945). Anarquista individualista y figura pionera del feminismo en Brasil, Lacerda de Moura fue una incansable propagandista, que dedicó muchos de sus escritos a campańas como el anticlericalismo, el antimilitarismo, el antifascismo, la pedagogía racionalista y antiautoritaria o la revolución social libertaria en general ${ }^{19}$. Destacó también en su defensa del neomaltusianismo, una nueva moral sexual, la emancipación de las mujeres o el pacifismo, aspectos todos ellos que la vincularon muy estrechamente a

18 Reseñas biográficas de todos estos colaboradores en Íñiguez (2008).

19 Moreira Leite (1984). 
Estudios. Sus textos, sobre todas las temáticas mencionadas, fueron recogidos en una gran cantidad de números de la revista, convirtiéndose en una de sus colaboradoras más habituales y de mayor recorrido en la historia de la publicación: un total de 36 artículos de la escritora brasileña entre 1931 y 1936. En febrero de 1931, el conocido escritor radical Santiago Valentí Camp ofrecía en Estudios un perfil de la autora y su obra como introducción a una serie de artículos de Lacerda de Moura que la revista publicaría a partir de entonces en sus páginas:

Los lectores de ESTUDIOS saborearán con deleite inesperado los sabrosos artículos de esta ilustre pensadora y tendrán ocasión de comprobar cuán pálido resulta este estudio frente a la realidad. Sería de desear, es más, sería preciso hacer lo posible para que los sustanciosos libros de María Lacerda fuesen muy pronto vertidos a nuestro idioma, en vez de traducirse cosas manidas y sin ninguna originalidad. Al divulgar en español los libros de esta escritora brasileña prestaríamos un servicio enorme a la humanidad y al mismo tiempo haríamos una obra de justicia para con esta autora vibrante y magnífica, casi desconocida hoy por el público hispano ${ }^{20}$.

Estudios fue la revista española que más textos publicó de la pensadora brasileña, entre ellos fragmentos de su ensayo Han Ryner e o amor no plural (1933). Lacerda de Moura era seguidora de este anarcoindividualista francés, seudónimo de Henri Ner, por otra parte uno de los grandes referentes ideológicos y culturales de la revista valenciana: Estudios publicó numerosos artículos de Ryner (además de textos de otros colaboradores de la revista sobre él, como los de la propia anarquista brasileña) y más de una treintena de sus cuentos en sus páginas.

Como señalábamos, los artículos de Lacerda de Moura en Estudios trataron múltiples cuestiones como el anticlericalismo, el antifascismo o la crítica social en general, aunque fueron la pedagogía y, sobre todo, la crítica a la moral sexual imperante, el neomaltusianismo y en especial la reflexión sobre la situación social de las mujeres desde una perspectiva anarcofeminista, los temas más habituales de estas colaboraciones. Lacerda de Moura consideraba

20 Valentí Camp, Santiago, "La pensadora María Lacerda de Moura», Estudios, 90, febrero 1931. Los artículos serían traducidos por el también conocido militante anarcoindividualista José Elizalde. Un sector del movimiento libertario español se interesó siempre por la obra de Lacerda de Moura y no la olvidó. En Cénit, revista cultural del exilio anarquista espańol, encontramos una semblanza biográfica de esta autora. Véase Cénit (Toulouse), 49, enero 1955. 
la maternidad consciente y el control de natalidad como elementos fundamentales de la emancipación femenina y resultan especialmente significativas algunas de sus críticas a aquellos que, aun dentro del movimiento libertario, no compartían la importancia de esos principios. En cierto sentido, sus quejas contra esa incomprensión recordaban las de la propia revista Estudios y las de los sectores anarquistas partidarios de estas ideas ante un mundo ácrata y sindicalista que mayoritariamente consideraba estas cuestiones secundarias cuando no contraproducentes en su proyecto revolucionario: «Para la reducida capacidad mental de los reaccionarios y de algunos revolucionarios que sustentan la teoría de la superpoblación, la mujer no es otra cosa que una máquina destinada a fabricar carne de cañón o de barricada. Para ellos no existe el problema femenino; consideran sencillamente a la mujer como un receptáculo de expansiones genésicas: la libertad, la Acracia que anhelan, es sólo para hombres ${ }^{21}$.

Otro tema de las colaboraciones en la revista de Lacerda de Moura, activa partidaria de la resistencia no violenta, fue la crítica de la violencia y de las guerras y la defensa del pacifismo y la fraternidad universal. Así, por ejemplo, la escritora brasileńa presentó en varios de sus artículos en Estudios la llamada Asociación Internacional Biocósmica, sociedad internacional «animada por el espíritu científico y filosófico, libre en absoluto de todo dogma» y formada por «sabios, biólogos, físicos, astrónomos, pensadores y filósofos, exonerados de todo sentimiento religioso o sectarista de las creencias, organizadas, laicas o no». Progresista, pacifista y universalista, ribeteada de pensamiento teosófico — del cual Lacerda de Moura, así como otros colaboradores de la revista (como el doctor Roberto Remartínez, por ejemplo) eran seguidores-, para la anarquista brasileña la Asociación tenía como principal objetivo «hacer desaparecer todas las causas de las guerras, para lo cual procura reforzar los lazos biocósmicos que inducen a los hombres a sentirse hermanos en la gran familia humana, solidarizándonos entre sí y con el "Cosmos increado" [...]. Aspira, asimismo, a reemplazar todas las religiones teístas, que dividen a los humanos en vez de unirles fraternalmente, por el principio vitalísimo de la «fraternidad universal $» »^{22}$

Pero Estudios no dio a conocer solo los textos de Lacerda de Moura a través de los números de la revista. También reseñó o dio cuenta —en su sección

21 Lacerda de Moura, Maria, «Procreación y miseria», Estudios, 106, junio 1932 (en cursiva en el original).

22 Lacerda de Moura, Maria, «La Asociación Internacional Biocósmica», Estudios, 118, junio 1933. La segunda parte de este artículo se publicó en el siguiente número de la revista (119, julio 1933). 
«Bibliografía»— de muchos de sus libros, publicados originalmente en portugués en Brasil, por lo general en la editorial Civilizaçao Brasileira de Rio do Janeiro, o en Editorial Paulista, A. Sementeira o Ediçoes Unitas, de Sao Paulo. Entre ellos: Civiliçao, tronco de escravos; Servicio militar obligatorio para a mulher? Recusa-mo! Denuncio!; A Mulher é uma degenerada?; el ya mencionado Han Ryner e o amor no plural; Clero e fascismo, horda de embrutecedores!, o Amai-vos e nao vos multipliqueis. Precisamente esta última obra, alegato de Lacerda de Moura en pro del neomaltusianismo, sería publicado por Estudios en su editorial: Amaos y no os multipliquéis, también con traducción de José Elizalde (Valencia, Biblioteca de Estudios), hacia 1937.

Múltiples puntos de conexión, por tanto, en temas además neurálgicos para la revista, de Estudios con la escritora anarcoindividualista brasileña, quien llegó a señalar asimismo en uno de los artículos aparecidos en la publicación valenciana, que Estudios era «la revista espańola que en la actualidad sostiene con mayor gallardía el pabellón del pensamiento libre» ${ }^{23}$.

En mayo de 1929, Estudios saludó la aparición de una revista de carácter específicamente naturista: Minerva $^{24}$, editada en Nueva York y dirigida por el escritor libertario venezolano Carlos Brandt. Al año siguiente (marzo de 1930) se anunciaba asimismo la salida del primer número de otra publicación mensual cultural, también publicada por Brandt en Nueva York: Palas. Revista Mensual Ilustrada, con una orientación asimismo naturista y que comenzaba desde su primer número con la publicación de un "Curso de naturopatía»:

PALAS, revista mensual ilustrada, número 1. Nueva York. Para encomiar la importancia de esta nueva publicación basta indicar el nombre de su director: Carlos Brandt. Revista de cultura física, intelectual y moral; lleva el sello inconfundible de la personalidad de su director. Naturismo, moral racional, sabiduría. El plan es excelente y tenemos la seguridad de que se desarrollará en todas sus partes. En este número inicial, muy bien confeccionado y muy bien redactado, comienza la publicación de un interesante Curso de Naturopatía, cuyo valor no es necesario encomiar. Reciba el simpático colega nuestra más cordial enhorabuena ${ }^{25}$.

Carlos Brandt (1875-1964) fue colaborador habitual de Generación Consciente y Estudios en gran variedad de temas, desde el naturismo y la

23 Lacerda de Moura, Maria, «El salvajismo fascista contra los israelitas», Estudios, 120, agosto 1933.

24 «Minerva. Revista mensual naturista», «Bibliografía», Estudios, 69, mayo 1929.

25 «Bibliografía», Estudios, 79, marzo 1930. 
medicina al anticlericalismo o el pensamiento anarquista, pasando por la historia, los perfiles de grandes autores y personajes célebres, la filosofía, la música o la literatura. La publicación consideraba a Brandt un «verdadero orientador del naturismo ${ }^{26}$. Asimismo, se anunciaron y comentaron algunos de sus libros en las páginas de Estudios, en clave siempre muy elogiosa. Así, por ejemplo, en mayo de 1929 (núm. 82), Estudios anunció en su sección «Bibliografía» su libro El fanatismo religioso («sobre las mentiras y contradicciones de la Biblia»), también publicado en Nueva York. Los pedidos debían hacerse a la editorial que había dado a luz el libro: la de la más importante publicación libertaria en español de aquella ciudad, la conocida Cultura Proletaria, que editaría también otros títulos del anarquista venezolano. Estudios seguiría publicitando El fanatismo religioso en números posteriores de la revista, así como otros títulos de Brandt, como La superstición médica, o sus diversas obras sobre naturismo o vegetarianismo - que, como comentábamos, Estudios consideraba de gran interés en ese ámbito— o sobre otras temáticas abordadas por el prolífico y polifacético escritor libertario venezolano.

La editorial de Generación Consciente y Estudios publicó asimismo dos de las obras de Brandt: Camino de perfección, en 1928, y La belleza de la mujer. Tratado de las proporciones armoniosas del cuerpo humano y de la importancia filosófica, artística y sociológica de la belleza física, en 1935 (libro originalmente editado en 1905), con ilustración de cubierta realizada por Manuel Monleón.

Carlos Brandt, activo propagandista del anarquismo, el naturismo, el vegetarianismo o el pacifismo, reunía un perfil ideológico e intelectual, por tanto, muy próximo a Estudios. Brandt vivió exiliado de Venezuela por razones políticas durante buena parte de los largos años de la dictadura de Juan Vicente Gómez, y ese carácter trashumante y cosmopolita conectaba también con el espíritu de la revista. Fue un viajero incansable y residió asimismo en Estados Unidos, especialmente en Nueva York, donde desarrolló una intensa actividad, como vemos. Durante ese período siguió manteniendo su vínculo con Estudios y poniendo en marcha iniciativas editoriales como las mencionadas, afines a aquella en orientación y temática.

En un tema central para Estudios como el neomaltusianismo y la difusión del control de la natalidad (en estrecha vinculación con la eugenesia y la reforma sexual), un colaborador habitual de la revista fue el médico, sociólogo, escritor y activo militante libertario argentino Juan Lazarte (18911963). Discípulo del pedagogo anarquista también argentino Julio Barcos (asimismo asiduo colaborador de Generación Consciente y Estudios, como mencionamos antes), fue el autor de un trabajo que publicó Estudios en siete

26 Estudios, 69, marzo 1929. 
entregas con el título general de «Significación cultural y ética de la limitación de los nacimientos", artículos que aparecieron entre los números 120 (agosto 1933) y 126 (febrero 1934). En ellos, Lazarte realizaba una vehemente y sólida defensa del neomaltusianismo, rastreando en sus precedentes históricos y situándolo como auténtico instrumento de liberación dentro de un programa general de transformación revolucionaria de la sociedad. Porque el control de natalidad, desvinculado de este propósito y aislado de la eugenesia — destinada a mejorar la calidad de la vida humana- y de la emancipación de las mujeres — «fisiológicamente» libres ahora, gracias a la contracepción-, en el contexto de una auténtica «revolución sexual», carecería de sentido. Todos estos elementos aparecen así estrechamente conectados, con el necesario concurso de una educación sexual que garantizara, entre otras cosas, la enseñanza y difusión de los distintos métodos anticonceptivos.

Lo apuntado por Lazarte coincidía en lo sustancial con lo defendido desde hacía tiempo por distintos redactores y colaboradores centrales de Estudios. No es de extrañar, por tanto, esta vinculación del médico libertario argentino con la revista valenciana. Contamos, además de con investigaciones que han profundizado en el pensamiento de Lazarte en torno a estos temas ${ }^{27}$, con un artículo que ha analizado específicamente esta colaboración de Lazarte en Estudios a cargo de Isabel Jiménez-Lucena y Jorge Molero-Mesa. Para estos autores, "existía una verdadera sintonía de la revista Estudios como espacio de discusión y Lazarte como profesor ${ }^{28}$. La obra de este fue asimismo divulgada por distintos colaboradores de Estudios, como la ya mencionada Maria Lacerda de Moura, Félix Martí Ibánez o Higinio Noja Ruiz. Así, desde Estudios:

[...] se presentaba a Lazarte como una autoridad en la elaboración de conocimientos que llevarían a la construcción de alternativas necesarias para el cambio del sistema social vigente, confiriéndosele un estatus de autoridad en la práctica discursiva anarcolibertaria que representaba la revista Estudios. De esta forma, la obra de Juan Lazarte publicada en Estudios jugó un relevante papel en la acción legitimadora y reivindicativa de la limitación de los nacimientos y de la liberación de las mujeres ${ }^{29}$.

27 Destacan en los últimos años los trabajos de Nadia Ledesma Prietto, con diferentes artículos en torno al tema y su reciente libro, producto de su tesis doctoral: Ledesma Prietto (2016).

28 Jiménez-Lucena y Molero-Mesa (2014).

29 Ibid. 
Además de publicar otros artículos de Lazarte en torno a estos temas en sus páginas, Estudios anunció, por ejemplo, su libro La revolución sexual de nuestro tiempo, publicado por la editorial de la revista Nervio en Buenos Aires en 1932, así como otras obras de Lazarte en torno a la universidad, la socialización de la medicina, etc. Lazarte estaba muy vinculado, por otro lado, al grupo Tierra y Libertad de Barcelona y a Diego Abad de Santillán, con quien colaboró estrechamente. En Barcelona se publicaron asimismo sus obras $E l$ control de natalidad (Barcelona, Maucci, ca. 1935) o La crisis mundial del capitalismo. Su significación histórica (Barcelona, Guilda de Amigos del Libro, ca. 1935). Este último libro fue publicado también por la Editorial de Tierra y Libertad, asimismo de Barcelona, que editó además La locura de las guerras. Sus causas (1933) y La revolución sexual de nuestro tiempo. Psicología y crisis del matrimonio (1933).

Por último, podemos mencionar al ingeniero y biólogo mexicano Alfonso Luis Herrera (1868-1942) ${ }^{30}$, que publicó numerosos artículos en la revista en torno a la creación artificial de vida o "plasmogenia», tal como Herrera bautizó a esta nueva ciencia creada por él. En estos textos, que aparecieron en Estudios desde sus primeros números hasta la desaparición final de la publicación, Herrera exponía las conclusiones de sus experimentos químicos en pos de la reproducción de células vivas. Se trata de una biología intensamente militante en su materialismo, en su cruzada laica y cientifista contra toda concepción religiosa acerca del origen de la vida y el mundo ${ }^{31}$ y en el rechazo a cualquier forma de espiritualismo transcendente. Sin embargo, la plasmogenia, según Herrera, no estaba exenta de un fin: la «suprema filantropía» de la ciencia para servir al interés más alto de la mejora de la vida humana y capaz de proporcionar suficientes recursos para su supervivencia; una humanidad así en continuo progreso y exenta de atavismos bélicos: «La plasmogenia es la suprema filantropía porque aspira a explicar nuestro origen para liberarnos del fanatismo; a fabricar los alimentos artificiales para impedir el hambre y los estragos de su aliada la guerra; a proclamar y probar que nadie nos espera después de la muerte, por qué somos productos exclusivamente químicos de las fuerzas naturales, ciegas e implacables ${ }^{32}$.

No fue esta la única editorial libertaria española de esos años con la que Herrera tuvo vinculación. El biólogo mexicano publicó también en la colección de folletos Cuadernos de Cultura de Valencia, que puso en marcha a

30 Aullet Bribiesca (2012).

31 Herrera, Alfonso L., "Conspiración clerical contra la plasmogenia: especial para Estudios (Anticlericalismo)», Estudios, 99, noviembre 1931.

32 Herrera, Alfonso L., «La sordera mística de la ciencia», Estudios, 130, junio 1934. 
inicios de la década de los treinta el teórico sindicalista valenciano Marín Civera, director asimismo de Orto. Revista de Documentación Social (19321934), una publicación con muchos puntos de contacto ideológicos y temáticos con Estudios ${ }^{33}$. En Cuadernos de Cultura, Herrera editó sus obras: La Plasmogenia, nueva ciencia del origen de la vida (1932) y El híbrido del hombre y el mono $(1933)^{34}$.

\section{REDES EDITORIALES}

Hemos contabilizado más de cuarenta referencias de publicaciones periódicas de diversos países americanos que fueron reseñadas por la revista en su sección «Bibliografía». Ello da buena cuenta de la red estructurada a través de mutuas informaciones y el intercambio de números entre todas estas cabeceras y Estudios. Muchas no tenían una impronta libertaria, pero coincidían en los propósitos librepensadores o progresistas o, incluso, en una ecléctica defensa de la difusión cultural entendida en sí misma como proyecto emancipador.

De nuevo destaca aquí Argentina. Pueden citarse, entre otras, las revistas culturales Nosotros (Revista Mensual de Letras, Arte, Historia, Filosofía y Ciencias Sociales); Claridad. Revista de Arte, Critica y Letras (Buenos Aires); Sendas Nuevas. Revista de Educación; Vida Nueva (naturista), o El Auto Rosarino. Revista de Difusión Cultural, dirigida por Gaston Leval (Rosario), por referenciar solo algunas. Mención especial merece una revista con muchos puntos de contacto ideológicos y temáticos con Estudios: Nervio. Ciencias, Artes, Letras (Buenos Aires, 1931-1936), desde cuyas páginas se calificaba a la revista valenciana, por cierto, como "excelente publicación ecléctica» ${ }^{35}$.

Mencionaremos solo algunos ejemplos de revistas de países citados con frecuencia por sus publicaciones. Es el caso de Uruguay (entre ellas La Pluma. Revista Mensual de Ciencias, Artes y Letras, El Hombre. Periódico Anarquista, de Montevideo) y Perú (La Senda. Revista ideológica, literaria, informativa y comercial, Juventud. Revista ilustrada de crítica social y extensión universitaria, de Lima). También Cuba, entre otras, y en especial Pro-Vida. Revista Naturista de La Habana, publicación dirigida por Aquilino López, que Estudios

33 Navarro Navarro (1997c y 2014) y Paniagua (2001). En este último volumen, cuya edición corre a cargo también de Javier Paniagua, se recoge una reproducción facsímil de esta publicación.

34 La editorial Somo de Barcelona editó también en 1930 su obra Dios ante la ciencia.

35 Nervio. Ciencias, Artes, Letras, 3, julio 1931. 
anunció en varios de sus números. Asimismo, aparecen referenciadas revistas de Chile, Ecuador, Costa Rica o Puerto Rico.

Significativamente, no aparece más que una mención a publicaciones mexicanas, aunque se haga repetidamente en este caso: Crisol. Revista de Crítica (México), reseñada en diversas ocasiones. Por el contrario, la alusión a Estados Unidos aparece en varias publicaciones, muy bien acogidas por Estudios, y corresponden en su mayoría a las ya mencionadas cabeceras puestas en marcha por el venezolano Carlos Brandt: Minerva: Revista Mensual Naturista y Palas, Revista Mensual Ilustrada (las dos de Nueva York) ${ }^{36}$.

Asimismo, se anunciaron títulos de libros y folletos publicados por numerosas editoriales americanas. Sobresale aquí la presencia de la literatura, aunque también los temas sociales. Centrándonos en estos últimos — haciendo referencia a las editoriales y entresacando solo algunos ejemplos de los libros reseñados-, puede destacarse de nuevo Argentina. Podemos citar, entre las más mencionadas, Ediciones Nervio, de Buenos Aires, vinculada con la publicación homónima aludida más arriba y tan cercana a Estudios (con títulos como La revolución social de nuestro tiempo, de Juan Lazarte; Camisas negras, de Luce Fabbri, o Todos ahora, contra la guerra, por J.Maguid). También de Buenos Aires, encontramos editoriales como Argos, Reja, Letras, Tor ( $\mathrm{La}$ herencia de un proletario, de A. de Carlo), Imán, Asociación Racionalista Judía o Claridad.

Por supuesto, aparecieron referencias a las ediciones libertarias argentinas de la célebre La Protesta (La revolución del Pueblo, de Joaquín Aznar). Precisamente, a finales de 1930 Estudios anunciaba que distribuiría títulos de esta editorial de la publicación más importante del anarquismo argentino, en concreto del folleto de Luis Bulffi ;Huelga de vientres! La revista valenciana se hacía eco del asalto que había sufrido el portavoz del movimiento libertario argentino en esas fechas por parte de la policía de la dictadura del general Uriburu, tras su golpe de Estado ese año ${ }^{37}$.

Fuera de Buenos Aires, otras editoriales argentinas reseñadas fueron Símbolo (El fanatismo religioso. Análisis crítico de la Biblia, del ya mencionado Carlos Brandt) o la Librería Ruiz, ambas de Rosario. En Chile encontramos referencias de las editoriales Nascimiento y la Imprenta Nos (Santiago) o de la Biblioteca Walton (Valparaíso). De Uruguay, las editoriales La Facultad,

36 Asimismo, en diciembre de 1929, Estudios (núm. 76) anunciaba la salida de otra publicación editada en Nueva York en español: Intuición. Revista Mensual de Ideas, Sociología y Crítica Constructiva, muy probablemente vinculada a la Librería Intuición de esa ciudad y ya mencionada más arriba.

37 Estudios, 88, diciembre 1930 
Mañana (que publicó Juan Cristóbal, de Romain Rolland) o Publicaciones Analectas (Teatro Infantil, por Albano Rosell), todas de Montevideo. De Ecuador, la editorial Labor; los Talleres Gráficos Nacionales, en Quito; Publicaciones de la Empresa Editorial Holguín y Castillo (La escuela para la vida, por la vida, de Polidoro Arellano) de Ambato, o Artes Gráficas Senefelder de Guayaquil. También aparece mención a Ediciones El Fígaro, de La Habana (Cuba), y la Editorial Elite, de Caracas (Venezuela).

En contraste con la escasa alusión a publicaciones periódicas mexicanas, las referencias a editoriales y a la producción de estas son abundantes en Estudios. Así, encontramos, entre otras, Ediciones de Crisol o la Editorial de la Federación de Escritores Proletarios (Barricada. Poema, de M. ${ }^{a}$ Luisa Vera y José Muñoz Costa; Cuadernos de literatura proletaria o Romances de la hoz y el martillo, poemas de José Muñoz Costa), o la Editorial Lumen (con un Bokabulario Esperanto-Ispano-Amerikano de Jesús Anaya).

\section{CONCLUSIONES}

El proyecto editorial de Generación Consciente y Estudios (revista y catálogo de libros y folletos) tuvo siempre, y ya desde sus inicios, una vocación transnacional, vinculándose a movimientos ideológicos, corrientes de pensamiento y debates internacionales durante el período de entreguerras. En primer lugar, por supuesto, y dado el origen ácrata de la publicación (aunque siempre con su orientación «ecléctica»), con las redes de militancia libertaria en esos años en Europa y América. Destacó en particular la influencia del anarcoindividualismo, sobre todo francés. Ya hemos mencionado el ascendiente sobre la revista de Han Ryner — de quien se publicaron numerosos artículos y cuentos, así como comentarios y reseñas sin fin sobre sus libros por parte de distintos colaboradores de Estudios-, pero también podríamos citar a Émile Armand (sobre cuyas tesis en torno a la "camaradería amorosa» se discutió ampliamente en la publicación) o anarcoindividualistas de otros países, como la ya comentada escritora brasileña Maria Lacerda de Moura o el pacifista y humanitarista rumano Eugen Relgis. La influencia de este anarquismo individualista fue muy notoria sobre Estudios en determinadas temáticas, como el pacifismo o la reforma sexual (con la difusión de artículos y obras de anarcoindividualistas franceses muy activos en esta campaña, como Manuel Devaldès, André Lorulot o Gérard de Lacaze-Duthiers, entre otros). Pero también muchos otros conocidos militantes del movimiento anarquista internacional colaboraron en sus páginas: Gaston Leval (seudónimo de Robert Pillar), Hem Day (Marcel Dieu), Léo Campion, G. Hardy (Gabriel Giroud), 
Camillo Berneri, Sébastien Faure, Hugo Treni (Ugo Fedeli), Eugène Humbert, Jeanne Humbert, G. Hardy (Gabriel Giroud), etc. —estos tres últimos también muy comprometidos defensores del neomaltusianismo y la eugenesia con fines revolucionarios-.

Uno de los objetivos principales de la revista y sus responsables fue asimismo conectarse estrechamente con el movimiento internacional (de Europa a América) por la reforma sexual, el control de la natalidad y la eugenesia, temas centrales en la publicación y que le otorgaban sus más importantes señas de identidad. En referencia a la eugenesia, uno de los principales colaboradores de la revista y difusor de estos planteamientos en Estudios — así como uno de sus introductores en general en España-, el maestro y pedagogo socialista Luis Huerta, señalaba en uno de sus artículos en torno a aquella:

No queremos que falte en este trabajo un especial homenaje de justa alabanza a los preclaros eugenistas iberoamericanos, hermanos de raza e ilustres amigos nuestros: doctor Víctor Delfino, de Buenos Aires; doctor Renato Kehl, de Río de Janeiro; doctor Carlos E. Pas Soldán, de Lima; profesor biólogo Alfonso L. Herrera y doctor Emiliano Torres, de México; profesor antropólogo Julio C. Salas, de Caracas, y los portugueses profesor antropólogo A. A. Méndez Correa, de Oporto, y profesor Cruz Filipe, de la Casa Pía de Lisboa. Su labor de pionniers cada día alcanza mayor auge, y ellos son los primeros luminares en la nebulosa resoluble de los pueblos libres de Iberoamérica hacia un futuro más sano, más rico y más consciente ${ }^{38}$.

En su campaña en pro de una nueva moral en lo que a la sexualidad se refiere, Estudios se vinculó al movimiento de reforma sexual europeo de entreguerras, por ejemplo, a la conocida Weltliga für Sexualreform (en inglés World League for Sexual Reform, y en español Liga Mundial para la Reforma Sexual sobre Bases Científicas), que animaban médicos progresistas y muy activos en estas campañas, como el alemán Magnus Hirschfeld o el británico Havelock Ellis. De hecho, el editor y director de Estudios, el alcoyano Joaquín Juan Pastor, y el médico ácrata alavés Isaac Puente (posiblemente la voz más influyente de la revista en estos temas y uno de sus más asiduos colaboradores) figuraron entre los adherentes españoles al congreso que dio origen a la Liga, en Copenhague en 1928. Estudios contribuyó a difundir las tesis de la Liga (que vinculaban también la reforma sexual al control de natalidad, la eugenesia, la educación sexual y la emancipación de las mujeres) en los círculos libertarios y obreristas de España y América, al igual que hizo otra publicación ecléctica

38 Huerta, Luis, «El maltusianismo no es el eugenismo», Estudios, 77, enero 1930. 
valenciana, la ya mencionada $O r t 0^{39}$. El programa de la Liga coincidía en lo sustancial con lo defendido por Estudios en este ámbito y por muchos de sus colaboradores europeos y americanos, aunque no obstante algunos de ellos, como el propio Isaac Puente, criticaron el carácter burgués y meramente social-reformista de la Liga, pese a compartir buena parte de sus objetivos concretos. Puente argumentaría finalmente en las propias páginas de la revista su rechazo a la invitación a formar parte de la sección española de la Liga en $1932^{40}$.

Asimismo, en Estudios colaboraron decenas de médicos y científicos de distintos países, no solo sobre estas cuestiones, sino también sobre muy distintos temas de divulgación médica y científica en general. El naturismo en particular fue otro de los ejes de atención de la revista ${ }^{41}$. Hemos mencionado el caso del venezolano Carlos Brandt, pero también podemos incluir aquí a los médicos más frecuentes colaboradores de Estudios y asimismo activos partidarios del naturismo, como Roberto Remartínez (titular de la sección de preguntas y respuestas médicas de la revista) o Isaac Puente. Asimismo, la publicación daba cuenta de la celebración de encuentros y congresos naturistas internacionales y de la actividad de la llamada Unión Naturista Internacional.

Estudios desarrolló también una intensa propaganda del pacifismo. Por un lado, desde la tradición histórica libertaria, recogiendo textos y artículos en este sentido de anarquistas europeos contemporáneos, fundamentalmente franceses como Han Ryner y los anarcoindividualistas mencionados más arriba (a los que habría que añadir a la brasileña Maria Lacerda de Moura), de otros anarcopacifistas como Hem Day o Léo Campion, así como de autores difícilmente clasificables como el humanitarista rumano Eugen Relgis, con numerosas colaboraciones en Estudios y muy alabado por la publicación. Por otro lado, habría que añadir la difusión por parte de la revista (en sus páginas o en el catálogo de su servicio de librería o su editorial) de los textos de los principales autores de la literatura progresista

39 Aquí la difusión de los principios y actividades de esta Liga fue mayor si cabe, dado el protagonismo en ella de algunos de sus más asiduos colaboradores, como la conocida Hildegart Rodríguez, Luis Huerta o el mismo director de Orto, Marín Civera, animadores de su sección española. Navarro Navarro (2014).

40 Puente, Isaac, "Carta abierta a la Liga Española para la Reforma Sexual sobre Bases Científicas», Estudios, 107, julio 1932. Entre otras cosas Puente señalaba que, de habérsele consultado para elegir el título de la asociación, él habría escogido el de Liga Española para la Subversión Sexual sobre Bases Humanas.

41 Sobre el naturismo libertario, Roselló (2003). 
pacifista del período de entreguerras (en la que la condena de los horrores de la Primera Guerra Mundial se unía a la denuncia del creciente militarismo y la amenaza belicista del fascismo en la década de los treinta), en forma de ensayos, artículos, cuentos y novelas, entre ellos Romain Rolland, Henri Barbusse, Ludwig Renn, Erich Maria Remarque, etc.

De manera creciente a lo largo de los años treinta, se multiplicaron en las páginas de la revista los artículos en contra de la guerra, las estadísticas, dibujos y fotos antibelicistas, e incluso apareció una sección específica: «Abajo la guerra». Asimismo, Estudios dio a conocer diferentes iniciativas pacifistas internacionales, con asociaciones como la War Resisters' International — con su sección española, Liga Española de Refractarios a la Guerra-, la Liga Internacional de Combatientes por la Paz, los Hogares Libres Internacionales, etc.; entidades que promovían en general la fraternidad universal, como la ya mencionada Asociación Internacional Biocósmica, presentada por Maria Lacerda de Moura, así como también iniciativas de partidarios de las lenguas planificadas como el esperanto o el ido, promovidas desde el anarquismo y donde el pacifismo tenía también un rol central.

En la geografía de la proyección y difusión de Estudios fuera de España, América ocupó un lugar prioritario. En primer lugar, económicamente, dado el volumen de ventas de la revista y de sus colecciones de libros y folletos en aquel continente; ello contribuyó sin duda a la supervivencia de Estudios. Esta vinculación ya venía desde los años de Generación Consciente, con diecinueve puntos de distribución fuera de la península: uno en las Islas Canarias, dos en Francia, trece en Latinoamérica y tres en Estados Unidos. América fue un ámbito de expansión de la publicación mucho más evidente y sensible para esta que otros países europeos, incluyendo Francia.

Por otro lado, y en cuanto a sus contenidos, la presencia de una amplia nómina de escritores de origen americano en las páginas de Estudios, de noticias e informaciones sobre América, así como de numerosas reseńas y anuncios de publicaciones periódicas «hermanas» o afines, o de editoriales de libros y folletos de allí, ratifican esta dimensión americana de la revista.

Otros factores reforzaron este vínculo transatlántico. No solo la evidente comunidad lingüística hispanohablante, los fuertes lazos entre conocidos militantes y núcleos libertarios a ambos lados del Atlántico o la presencia de grupos de inmigrantes de origen español y de militantes ácratas ibéricos trashumantes que allí residían y que escribían regularmente en Estudios (como los ya mencionados Costa Iscar, Campio Carpio, José Martínez Novella o el francés Gaston Leval, tan vinculado a España y a Argentina). También cabe hacer referencia a la presencia de sectores del movimiento obrero y grupos de anarquistas y librepensadores lectores de la revista en estos países e influidos por ella. Y especialmente 
a la existencia de un bien nutrido grupo de médicos, escritores, científicos o militantes, propagandistas y activistas (y en ocasiones todas estas cosas a la vez) en el continente americano que colaboraron en las páginas de la revista o en su catálogo editorial y con los que Estudios estableció sólidos vínculos.

El punto de conexión fue en este último caso no solo la sintonía ideológica (ácrata, anarcosindicalista, obrerista, radical o librepensadora), sino también el hecho de compartir buena parte de las campañas defendidas por Estudios. Nos hemos referido aquí en particular a temas centrales de la revista, como la reforma sexual y la difusión de los planteamientos neomaltusianos y eugenésicos, el naturismo o el pacifismo, y la aportación clave en estas campañas de médicos, científicos, escritores y activistas americanos, de los cuales hemos escogido solo algunos ejemplos, como fue el caso de Maria Lacerda de Moura, Carlos Brandt, Juan Lazarte o Alfonso L. Herrera.

A través de la divulgación del pensamiento y la obra de todos ellos, a la vez que paralelamente se difundían los textos, opiniones e iniciativas de los movimientos europeos internacionales contemporáneos alrededor de todas estas cuestiones, desde una clave progresista y/o revolucionaria, y de sus principales representantes, Estudios actuó en buena medida como puente entre ambos continentes y contribuyó a difundir en su público lector de ambos lados del Atlántico los temas y las tesis afines a la revista, dentro del espectro ideológico ácrata (militantes, grupos, activistas). El objetivo de Estudios era, entre otras cosas, insertar definitivamente en el universo cultural anarquista estas cuestiones, entre las cuales asuntos como la reforma sexual, el control de natalidad o la eugenesia eran sin duda los más polémicos y de más difícil aceptación en estos medios. Sin olvidar por supuesto otros temas dentro de esta tradición ideológica, y dada la importancia teórica y práctica que la revista concedió a esa dimensión internacional, Generación Consciente y Estudios contribuyeron, sin duda, a consolidar una cultura libertaria transatlántica renovada en el período de entreguerras.

Pero la revista difundió todas estas actitudes y opiniones también de manera genérica en el ámbito obrerista, progresista y del librepensamiento español y latinoamericano. La revista reforzaba así una red que incluía propagandistas europeos y americanos de estas ideas e iniciativas, publicaciones periódicas afines o editoriales de libros y folletos, promoviendo un debate de dimensión internacional en torno a aquellas cuestiones. No se trataba de una mera divulgación neutra de novedades científicas. Neomaltusianismo, eugenesia, reforma sexual, naturismo, pacifismo o difusión científica eran reapropiados, reinterpretados y resignificados siempre desde una perspectiva que los integrara como instrumentos válidos a la vez que necesarios de un proyecto global de transformación social revolucionaria. 


\section{Bibliografía}

Álvarez Junco, J. (1991) [1976]. La ideología politica del anarquismo español (1868-1910). Madrid: Siglo xxi.

Álvarez Peláez, R. (1995). Eugenesia y darwinismo social en el pensamiento anarquista. En B. Hofmann, P. Joan i Tous y M.Tietz (eds). El anarquismo español y sus tradiciones culturales (pp. 29-40). Frankfurt; Madrid: Vervuert; Iberoamericana.

- (2011). Literatura sobre sexo en la España de los años veinte y treinta del siglo xx: entre medicina y pornografía. En J. L. Guereña (ed.). La sexualidad en la España contemporánea (1800-1950) (pp. 149-162). Cádiz: Universidad de Cádiz.

Aullet Bribiesca, G. (2012). Trascendencia del pensamiento y la obra de Alfonso L. Herrera. Historia Mexicana. 61 (4) (244), 1525-1581.

Cano Carrillo, S. (1972). Hombres del movimiento libertario. Higinio Noja Ruiz. Cénit. Revista Bimensual de Sociología, Ciencia y Literatura, 201, 5698-5700.

Cleminson, R. (2008). Anarquismo y sexualidad (España, 1900-1939). Cádiz: Universidad de Cádiz.

Díez, X. (2007). El anarquismo individualista en España (1923-1938). Barcelona: Virus.

Guereña, J. L. (2013). Les espagnols et le sexe. XIXe-Xxe siècles. Rennes: Presses Universitaires de Rennes.

Íñiguez, M. (2008). Enciclopedia histórica del anarquismo español. Vitoria: Asociación Isaac Puente.

Jiménez-Lucena, I. y Molero-Mesa, J. (2014). Una lógica desestabilizadora del orden social y sexual: el médico argentino Juan Lazarte en la revista anarquista Estudios (1932-1936). Asclepio. Revista de Historia de la Medicina y de la Ciencia, 66 (2). Disponible en: http:// dx.doi.org/10.3989/asclepio.2014.20.

Ledesma Prietto, N. (2016). "La revolución sexual de nuestro tiempo»: el discurso médico anarquista sobre el control de la natalidad, la maternidad y el placer sexual. Argentina, 19311951. Buenos Aires: Biblos.

Litvak, L. (1981). Musa libertaria. Arte, literatura y vida cultural del anarquismo español (1880-1913). Barcelona: Antoni Bosch.

Martí Ibáńez, F. (1975). Consultorio psíquico-sexual. Barcelona: Tusquets

Masjuan, E. (2000). La ecología humana en el anarquismo ibérico. Urbanismo «orgánico» o ecológico, neomalthusianismo y naturismo social. Barcelona: Icaria.

Moreira Leite, M. L. (1984). Outra Face do Feminismo: Maria Lacerda de Moura. Sao Paulo: Ática.

Nash, M. (1995). La reforma sexual en el anarquismo español. En B. Hofmann, P. Joan i Tous y M. Tietz (eds). El anarquismo español y sus tradiciones culturales (pp. 281-296). Frankfurt; Madrid: Vervuert Iberoamericana.

- (1999). Rojas. Las mujeres republicanas en la guerra civil. Madrid: Taurus.

Navarro Monerris, J. (1988). Generación Consciente. Sexualidad y control de natalidad en la cultura revolucionaria española [trabajo de investigación inédito presentado al Instituto Juan Gil-Albert de Alicante].

Navarro Navarro, J. (1997a). El paraíso de la razón. La revista Estudios (1928-1937) y el mundo cultural anarquista. Valencia: Alfons el Magnànim. 
- (1997b): Anarquismo y neomalthusianismo: la revista Generación Consciente (1923-1928). Arbor, 156, 615, 9-23. Disponible en: https://doi.org/10.3989/arbor.1997.i615.1847.

(1997c): El camino hacia una cultura obrera de síntesis durante la II República: la revista Orto (1932-1934). Bulletin d'Histoire Contemporaine de l'Espagne, 26, 535-551.

- (2010). Los educadores del pueblo y la «revolución interior». La cultura anarquista en Espańa. En J. Casanova (ed.). Tierra y Libertad. Cien años de anarquismo en España (pp. 191-217). Barcelona: Crítica.

- (2014). Sexualidad, reproducción y cultura obrera revolucionaria en España: la revista Orto (1932-1934). Arbor. Ciencia. Pensamiento y Cultura. 190 (769). Disponible en: http://dx.doi.org/10.3989/arbor.2014.769n5014.

Paniagua, J. (2001). Marín Civera y la cultura popular. Orto, 1932-1934. En Orto (19321934). Revista de Documentación Social (pp. 15-58). Alzira, Valencia: Universidad Nacional de Educación a Distancia.

Roselló, J. M. (2003). La vuelta a la naturaleza. El pensamiento naturista hispano (1890-2000): naturismo libertario, trofologia, vegetarismo naturista, vegetarismo social y librecultura. Barcelona: Virus.

Shapiro, A. (1933). Informe sobre la crisis de la CNT. Recogido en: Estudios de Historia Social, 5-6, abril-septiembre 1978.

Sueiro Seoane, S. (ed.) (2013). Dossier «Las redes anarquistas transnacionales entre los siglos XIX y XX». Alcores. Revista de Historia Contemporánea, 15, 13-155. 